\title{
Quantification of myelin loss in frontal lobe white matter in vascular dementia, Alzheimer's disease, and dementia with Lewy bodies
}

\author{
Masafumi Ihara $\cdot$ Tuomo M. Polvikoski $\cdot$ Ros Hall $\cdot$ Janet Y. Slade $\cdot$ Robert H. Perry $\cdot$ \\ Arthur E. Oakley $\cdot$ Elisabet Englund · John T. O'Brien $\cdot$ Paul G. Ince $\cdot$ Raj N. Kalaria
}

Received: 16 March 2009/Revised: 24 December 2009/Accepted: 25 December 2009/Published online: 21 January 2010

(C) The Author(s) 2010. This article is published with open access at Springerlink.com

\begin{abstract}
The aim of this study was to characterize myelin loss as one of the features of white matter abnormalities across three common dementing disorders. We evaluated post-mortem brain tissue from frontal and temporal lobes from 20 vascular dementia (VaD), 19 Alzheimer's disease (AD) and 31 dementia with Lewy bodies (DLB) cases and 12 comparable age controls. Images of sections stained with conventional luxol fast blue were analysed to estimate myelin attenuation by optical density. Serial adjacent sections were then immunostained for degraded myelin basic protein (dMBP) and the mean percentage area containing dMBP (\% dMBP) was determined as an indicator of myelin degeneration. We further assessed the relationship between dMBP and glutathione $S$-transferase (a marker of mature oligodendrocytes) immunoreactivities. Pathological diagnosis significantly affected the frontal but not temporal lobe myelin attenuation: myelin density was most reduced in $\mathrm{VaD}$ compared to $\mathrm{AD}$ and $\mathrm{DLB}$, which still significantly
\end{abstract}

M. Ihara ( $₫) \cdot$ T. M. Polvikoski · R. Hall ·

J. Y. Slade - R. H. Perry · A. E. Oakley ·

J. T. O'Brien · R. N. Kalaria $(\square)$

Institute for Ageing and Health, Wolfson Research Centre,

Campus for Ageing and Vitality, Newcastle University,

Newcastle upon Tyne NE4 5PL, UK

e-mail: ihara@kuhp.kyoto-u.ac.jp

R. N. Kalaria

e-mail:r.n.kalaria@ncl.ac.uk

E. Englund

Division of Neuropathology, Department of Pathology,

University Hospital, Lund, Sweden

P. G. Ince

The Academic Unit of Pathology, Medical School, University of Sheffield, Beech Hill Road,

Sheffield S10 2RX, UK exhibited lower myelin density compared to ageing controls. Consistent with this, the degree of myelin loss was correlated with greater \%dMBP, with the highest \%dMBP in $\mathrm{VaD}$ compared to the other groups. The \%dMBP was inversely correlated with the mean size of oligodendrocytes in $\mathrm{VaD}$, whereas it was positively correlated with their density in AD. A two-tier regression model analysis confirmed that the type of disorder ( $\mathrm{VaD}$ or $\mathrm{AD}$ ) determines the relationship between \%dMBP and the size or density of oligodendrocytes across the cases. Our findings, attested by the use of three markers, suggest that myelin loss may evolve in parallel with shrunken oligodendrocytes in $\mathrm{VaD}$ but their increased density in $\mathrm{AD}$, highlighting partially different mechanisms are associated with myelin degeneration, which could originate from hypoxic-ischaemic damage to oligodendrocytes in $\mathrm{VaD}$ whereas secondary to axonal degeneration in $\mathrm{AD}$.

Keywords Alzheimer's disease - Myelin - Myelin basic protein - Oligodendrocyte - White matter - Vascular dementia

\section{Introduction}

The pathogenesis of white matter (WM) changes is unclear but they frequently although not always coincide with cerebrovascular risk factors such as hypertension, atherosclerosis and diabetes as is seen in patients with vascular dementia (VaD) [29]. Disturbances in cerebrospinal fluid production, disruption in blood-brain barrier permeability to macromolecules, and cerebral oedema [23, 38] have been cited as important causes in the development of WM changes. WM attenuation is also frequently observed in neurodegenerative disorders such as Alzheimer's disease 
(AD) [42] and dementia with Lewy bodies (DLB) [31]. On magnetic resonance (MR) imaging, such changes are apparent as WM lesions which increase with older age [18] and particularly so in AD and DLB compared to ageing controls, though to a lesser extent than in $\mathrm{VaD}$ [5]. Quantitative post-mortem MR imaging also reflects the severity of the neuropathological changes in the WM [21]. Recent diffusion tensor imaging studies suggest that the WM microstructure disintegrates with age, in particular, fibre populations within prefrontal region and internal capsule [6, 41].

The WM may be affected by hypoxic-ischaemic damage or alternatively by secondary demyelination subsequent to axonal degeneration due to neuronal damage [15]. A previous clinicopathological study indicated that compared to $\mathrm{AD}$, patients with $\mathrm{VaD}$ showed widespread diffuse changes, including spongiosis and arteriolosclerosis, along with état criblé and myelin loss [16], suggesting different etiologies of the WM damage between $\mathrm{VaD}$ and $\mathrm{AD}$. However, given the widely accepted fact that $\mathrm{VaD}$ and AD both increase in prevalence with age, frequently occur concomitantly, and overlap considerably in their symptomatology, pathophysiology, and comorbidity [25, 26], the WM changes in AD and even DLB may be caused by related vascular mechanisms. Therefore, our aim here was to quantify the severity of WM myelin loss and determine the underlying aetiology of $\mathrm{WM}$ abnormalities in $\mathrm{VaD}$ compared to AD and DLB.

To compare WM changes between controls and $\mathrm{VaD}$, $\mathrm{AD}$, and $\mathrm{DLB}$ cases, we used hematoxylin and eosin (H\&E) and luxol fast blue (LFB) staining and immunohistochemistry with antibodies against degraded myelin basic protein (dMBP). In the case of LFB, we developed a computer-assisted morphometric image analysis to quantify myelin attenuation in the WM. dMBP immunohistochemistry provided an index of WM damage because the antibody to $\mathrm{dMBP}$ recognizes a pathologically exposed epitope of the human MBP (an 81-87 amino acid sequence; QDENPVV) in demyelinating diseases such as multiple sclerosis [34], diffuse WM changes [47], and focal ischaemic damage (Tomimoto $\mathrm{H}$, unpublished data, 2008). To assess oligodendrocyte status within the disease groups, we immunostained selected cases with an antibody against glutathione $S$-transferase pi (GSTpi; a marker of oligodendrocytes) to evaluate if there was a relationship between oligodendrocytic change and WM damage. Previous studies have thus far reported decreases in oligodendrocytes in $\mathrm{VaD}$ [1] and in $\mathrm{AD}[42,43]$, although robust quantitative analysis in a larger series is lacking. Therefore in this study, we evaluated the integrity of a WM component and oligodendrocytes using novel markers to address whether the underlying mechanisms affecting the WM are similar among the three dementias.

\section{Materials and methods}

Subjects, histopathological and immunohistochemical procedures

Table 1 provides the demographics and the pathological features of the subjects. All the patients were prospectively assessed in Newcastle and the duration of clinical disease ranged between 6 and 12 years. We obtained coronal blocks of tissue from the frontal and temporal lobes from the Newcastle Brain Tissue Resource (NBTR) and excluded cases with pathological diagnoses of co-existing $\mathrm{VaD}$ and $\mathrm{AD}$ or $\mathrm{VaD}$ and $\mathrm{DLB}$. Thus, 82 cases were included in this study to select cases for relatively single processes, which consisted of 12 controls, 31 DLB cases, 19 AD cases, and $20 \mathrm{VaD}$ cases. Autopsies were performed within $12-36 \mathrm{~h}$ after death. The post-mortem intervals between death and tissue retrieval were not different between the groups. There were also no differences in the length of fixation of tissue in formalin between the cases and controls Brains were fixed for 4-6 weeks. As previously described [30], the neuropathological diagnoses of $\mathrm{VaD}$ [28], AD [9, 37], and DLB [35] were made by thorough histopathological examination of extensively sampled brain sections. The clinical study had ethical approval from the local Newcastle Ethics committees and all participants gave written consent to brain tissue donation. Use of brain tissue was also approved by the local research ethics committee of the Newcastle upon Tyne Hospitals NHS Foundation Trust and the NBTR committee.

Ten and $20-\mu \mathrm{m}$ thick paraffin-embedded tissue sections were cut from the frontal lobe at the level of the olfactory bulbs and the temporal lobe at the level of the anterior hippocampus and stained for H\&E and LFB $(20 \mu \mathrm{m})$ and for immunohistochemistry $(10 \mu \mathrm{m})$. To minimize variability in LFB staining and immunohistochemical intensity, control sections were included, tissue sections were prepared by the same technician and stained with or using freshly prepared tinctorial and buffer solutions.

\section{Assessment of myelin density}

Myelin density was determined in a large series of LFBstained serial coronal sections from cases and controls to select representative regions of interest (ROI) for analyses. The analyses were performed essentially as previously described [51]. To determine the myelin index, the WM was outlined automatically using the wand tool. When the border between the neocortex and the WM was obscured due to severe myelin loss, the WM was outlined manually using the semiautomatic trace tool instead of the wand tool. Any incidental border region of the cortex mixed in the outlined WM area was excluded from the analysis (Fig. 1, asterisk). 
Table 1 Age, gender and disease severity of the groups

\begin{tabular}{|c|c|c|c|c|}
\hline Parameter & Controls & DLB & $\mathrm{AD}$ & $\mathrm{VaD}$ \\
\hline \multicolumn{5}{|l|}{ Demographic details } \\
\hline$N$ & 12 & 31 & 19 & 20 \\
\hline Age $( \pm \mathrm{SD})$ & $75.6 \pm 7.8$ & $79.1 \pm 5.7$ & $85.5 \pm 5.5$ & $81.0 \pm 8.5$ \\
\hline Age range (years) & $61-92$ & $64-89$ & $75-96$ & $67-93$ \\
\hline Gender $(\mathrm{M} / \mathrm{F})$ & $6 / 6$ & $14 / 17$ & $7 / 12$ & $10 / 10$ \\
\hline \multicolumn{5}{|l|}{ Pathological features } \\
\hline Mean Braak stage & $1.0 \pm 1.0$ & $2.3 \pm 1.0$ & $4.9 \pm 0.8$ & $1.3 \pm 0.7$ \\
\hline Neuritic plaque density ${ }^{\mathrm{a}}\left(\right.$ per $\mathrm{mm}^{2}$ ) & $2 \pm 1$ & $21 \pm 3$ & $27 \pm 4$ & $7 \pm 2$ \\
\hline Lewy body pathology (ubiquitin score) & $<1$ & $7.2 \pm 2.6$ & $<1$ & $<1$ \\
\hline Lewy body pathology ( $\alpha$-synuclein score) & $<1$ & $12.5 \pm 5.0$ & $<1$ & $<1$ \\
\hline Number of vascular lesions (range) & $0-1$ & $0-3$ & $0-3$ & $3-15$ \\
\hline Degree of CAA (range) & $0-1$ & $0-4$ & $1-4$ & $0-4$ \\
\hline Frontal myelin index* & $51.1 \pm 9.5$ & $41.5 \pm 8.5$ & $35.9 \pm 11.1$ & $26.5 \pm 9.0$ \\
\hline Temporal myelin index & $37.3 \pm 10.1$ & $32.7 \pm 13.4$ & $35.2 \pm 11.0$ & $34.4 \pm 11.9$ \\
\hline
\end{tabular}

Unless otherwise stated numbers show mean values with standard deviation $( \pm \mathrm{SD})$. AD subjects were significantly older than controls and DLB $(P<0.01)$. Selected cases stained for amyloid $\beta$ protein immunoreactivity also showed the severity of neuritic plaque burden in AD and DLB was comparable to the phases 4 and 5 staging described by Thal et al. [46] and the BrainNet Europe Consortium [3]. The presence of Lewy body pathology was determined by ubiquitin and $\alpha$-synuclein immunostaining [35]. The number of vascular lesions and degree of CAA were recorded according to the Vonsattal grading as previously described $[28,40]$

* Significant difference $(P<0.05)$ between any two groups

${ }^{a}$ Neuritic plaque densities were comparable to the CERAD ratings in that moderate to severe scores were evident in $95 \%$ of $\mathrm{AD}$ and $74 \%$ of DLB cases

Fig. 1 Quartile analysis of LFB-stained section from the frontal lobe of a VaD patient (74 years). The WM was outlined in green and the grey levels within the WM were divided into four quartiles. The area percentage (\%area) for each quartile was calculated (labelled in red). Myelin index was then calculated for each case and control (see "Methods") (scale bar $1 \mathrm{~cm}$ )
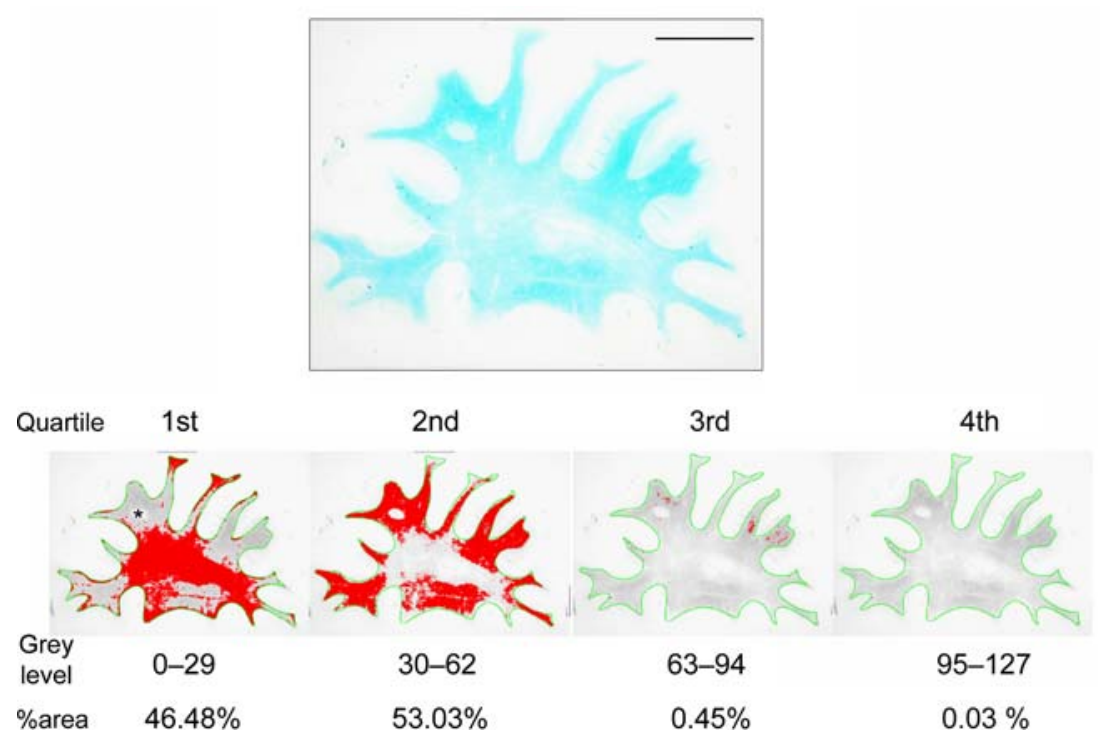

The detected range of grey levels within the WM, corresponding to the staining intensity, from the point $0-127$ (0, white; 255 , black) was divided into four quartiles (the first quartile 0-29, the second 30-62, the third 63-94, and the fourth $95-127)$. The percent area (\%area) for each quartile was calculated. The median grey level of each quartile $(14.5,46.0,78.5$, and 111.0), an estimate for the staining intensity, was then multiplied by \%area/100 in each quartile, which gave the total myelin index [51]. Braak staging [8] and plaque burden scores were used to explore the relationship with the myelin index. Chi-square analysis was performed to determine if higher Braak stage ( $\geq$ IV) affected myelin index (cut-off score $=$ median value of myelin index, 44.0 for the frontal lobe; 40.0 for the temporal lobe) or amyloid score (none/sparse or moderate/severe) in the combined cases of AD and DLB (28 cases with Braak 
stage 0 through III and 22 cases with Braak stage IV through VI). The H\&E-stained slides were examined in parallel but independently of the findings in the LFB images or case diagnosis. The WM damage was categorized as one of the following four grades as previously described [43] and corroborated by quantitative biochemical data [14]: (1) normal, normal WM; (2) mild, no appreciable reduction in axonal meshwork density and easily recognized long axons with occasional axonal debris and a slightly increased number of reactive astrocytes; (3) moderate, a slight reduction of axonal meshwork density and a reduction of oligodendroglial cell nuclei and a further increased number of reactive astrocytes; and (4) severe, a marked reduction of myelin, axons and oligodendroglial cell nuclei with relatively marked astrocytic reaction and loosely scattered macrophages, but no complete cerebral infarct. When several grades were observed in one section, the dominant grade represented the section.

\section{Assessment of dMBP staining}

Frontal sections from 59 cases (controls $n=8$, DLB $n=18, \quad \mathrm{AD} \quad n=14, \quad \mathrm{VaD} \quad n=19) \quad$ with $\mathrm{WM}$ area $>15 \mathrm{~mm}^{2}$ were stained with rabbit anti-dMBP sera (AB5864, Millipore, Billerica, MA, USA) essentially as previously described [51] using the Vectastain ABC System [27]. Images of 15-22 randomly selected ROIs in the WM were captured and then analysed [51]. The percentage immunostained area was measured within each field, which was averaged to yield \%dMBP for all the sections. Infarcted areas were always excluded from the analysis because of the entirely different patterns of dMBP staining. All of the above analyses were performed blind to the diagnosis by labelling the sections with arbitrary numbers.

\section{Assessment of GSTpi staining}

Serial sections from five cases of $\mathrm{VaD}$ and $\mathrm{AD}$ each and three cases of DLB immediately adjacent to those stained for dMBP were stained with an antibody against GSTpi (Thermo Fisher Scientific Anatomical Pathology, Cheshire, UK) to determine the density (number of oligodendrocytes $\left./ \mathrm{mm}^{2}\right)$ and the mean size $\left(\mu \mathrm{m}^{2}\right)$ of oligodendrocytes. For such staining, 15-22 images of the ROI as for dMBP staining were similarly captured and analysed using computer-assisted analysis. Our preliminary data suggested that staining quality exponentially diminished with prolonged postmortem delay, especially when it exceeded $30 \mathrm{~h}$. Some of the sections showed high background and reduced contrast, making it difficult to correctly identify oligodendrocytes. However, all cells with stained area of $4.2-25.3 \mu \mathrm{m}^{2}(40-240$ pixels) were counted after testing for accuracy for oligodendrocyte size rather than macrophages or other cells. Non-specific reactivities such as in blood vessel walls, mostly linear in shape with major axis larger than 24 pixels and minor axis smaller than 6 pixels, and macrophages were distinguished from the oligodendrocytic staining and automatically excluded from the analysis. In addition to GSTpi, we tested with success a range of other antibodies to detect mature oligodendrocytes including those to $2^{\prime}, 3^{\prime}$-cyclic nucleotide 3'-phosphodiesterase (clone 11-5b, SigmaAldrich, St. Louis, MO, USA), myelin oligodendrocyte glycoprotein (courtesy of Prof Richard Reynolds, Imperial College), Nogo-A (Abcam, Cambridge, UK), OLIG2 (Abcam) and SRY-box containing gene 10, SOX10 (clone 20B7, R\&D Systems, Abingdon, UK).

Statistical analysis

Statistical analysis was performed using StatView5.0 (SAS Institute, Cary, NC, USA). Frontal and temporal myelin densities were analysed separately. The \%area for the first or second quartile, the myelin index, and \%dMBP were compared among the four groups by an unpaired $t$ test. Frontal and temporal WM were analysed separately for the $\%$ area and myelin index. Pearson correlation analysis was performed to observe possible correlation of \% dMBP with the \%area for the first or second quartile, with myelin index, or with the size/density of oligodendrocytes. Chisquare analysis was performed to determine any correlation of myelin index with the Braak stage or plaque density in the AD and DLB cases combined (all except one DLB case showed Braak stage $\geq$ I). A two-level regression model was also applied to the hierarchical data set, with size or density of oligodendrocytes as a single outcome and $\% \mathrm{dMBP}$ as a response variable that was measured at the ROI (lower) level, and with age, gender, disease (disease code; $0=\mathrm{AD}$ and $1=\mathrm{VaD})$, and mean $\% \mathrm{dMBP}$ as explanatory variables at the case (higher) level using the HLM6 software. For all analyses, the level of statistical significance was set at $P<0.05$.

\section{Results}

Correlation between myelin index in LFB and WM grading by $\mathrm{H} \& \mathrm{E}$

The myelin indices determined from LFB-stained sections were grouped according to the four grades (normal, mild, moderate and severe) of WM damage indicated by H\&E $(n=35)$. The myelin index decreased with increasing severity of the WM pathology (Fig. 2). These data showed remarkable consistency in the assessment of WM damage between LFB and H\&E in the subset of cases. 


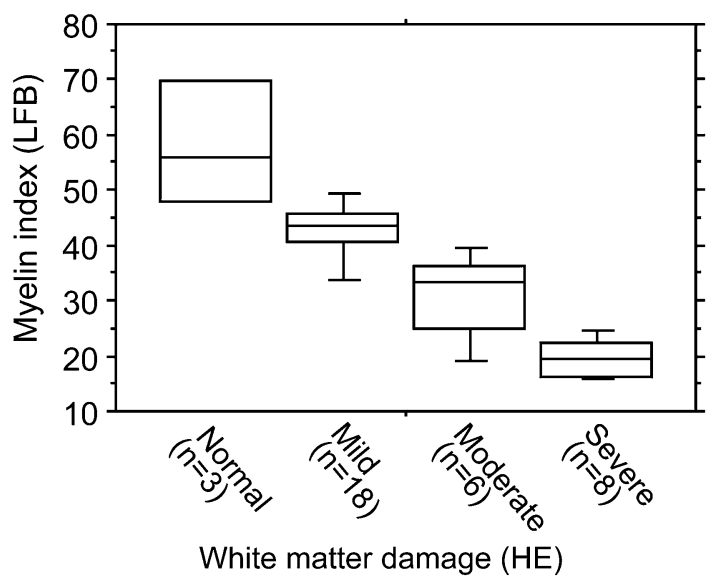

Fig. 2 Boxplot analysis for frontal myelin indices from LFB-stained sections in cases with microscopically evaluated normal WM $(n=3)$ and in those with WM changes categorized as mild $(n=18)$, moderate $(n=6)$, and severe $(n=8)$ in H\&E. The line within the box indicates the median value. Error bars are drawn at the $5 \%$ and the $95 \%$ confidence intervals

Myelin loss in the frontal and temporal lobes in dementing disorders as assessed by LFB staining

The diagnosis significantly affected the frontal myelin loss as assessed by the myelin index (Fig. 3; Table 1). The $\%$ area that fell into the first quartile (most severe myelin loss) increased with the following order: controls $(4.3 \%)<\mathrm{DLB}(18.3 \%)<\mathrm{AD}(34.6 \%)<\mathrm{VaD}(63.1 \%)$ (Fig. 3b). There were significant differences between any two groups except for the marginal significance between controls and DLB. Similarly, the frontal myelin index was significantly different between any two groups with the following order: controls $>\mathrm{DLB}>\mathrm{AD}>\mathrm{VaD}$ (Table 1; Fig. 3c), indicating that myelin loss was greatest in the $\mathrm{VaD}$ group. Age did not correlate with the myelin index $(r=-0.20$ for frontal WM; $r=-0.05$ for temporal WM). Chi-square test showed that higher Braak stage (IV, V, and VI) was significantly related to the lower frontal myelin index (less than 44.0) in the AD and DLB cases combined $(P=0.048)$. However, there were no clear correlations between plaque densities and myelin index.

By contrast, in the temporal lobe, the \%area for the first quartile was neither significantly different between any of groups (controls, 33.7\%; DLB, 47.4\%; $\mathrm{AD}, 38.1 \%$; and $\mathrm{VaD}, 41.2 \%$ ) nor was the temporal myelin index (Table 1), although the three dementia groups showed slightly more WM changes than controls. Chi-square analysis showed no significant association between the Braak stage and temporal myelin index (less than 40.0) in the AD and DLB cases combined $(P=0.21)$. There was also no significant correlation between frontal and temporal myelin index in any single group $(P=0.25$ for controls, $P=0.23$ for
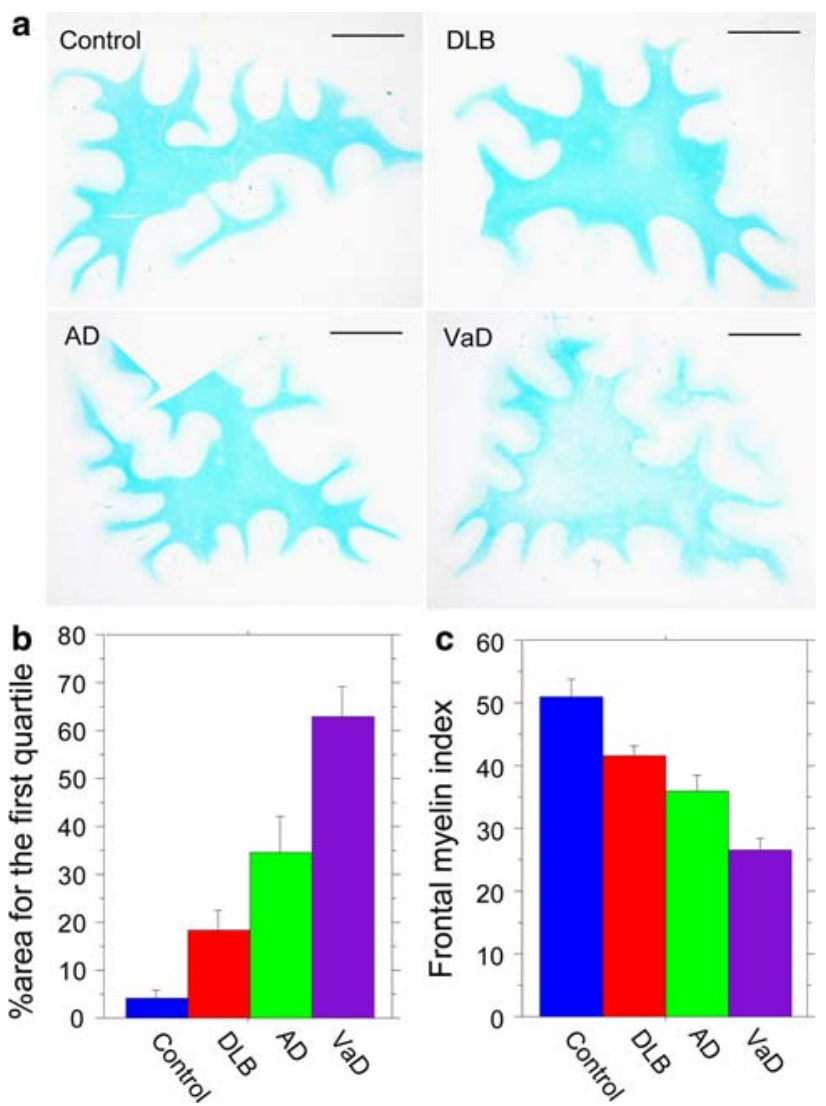

Fig. 3 Myelin loss in the frontal lobes in dementing disorders as assessed by LFB staining. a Representative images of LFB staining in cases of control (89 years), AD (87 years), DLB (77 years), and VaD (78 years) (scale bars $1 \mathrm{~cm}$ ). b The \%area for the first quartile in the four groups (Table 1). Differences were significant between any two groups except for that between controls and DLB; controls versus DLB, $P=0.0537$; controls versus $\mathrm{AD}, P=0.0032$; controls versus $\mathrm{VaD}, P<0.0001$; DLB versus $\mathrm{AD}, P=0.0464$; DLB versus $\mathrm{VaD}$, $P<0.0001 ; \mathrm{AD}$ versus $\mathrm{VaD}, P=0.0045$. $\mathrm{c}$ The frontal myelin index in the four groups. Differences were significant between any two groups: controls versus DLB, $P=0.0027$; controls versus $\mathrm{AD}$, $P=0.0005$; controls versus $\mathrm{VaD}, P<0.0001$; $\mathrm{DLB}$ versus $\mathrm{AD}$, $P=0.0473 ;$ DLB versus $\mathrm{VaD}, P<0.0001 ; \mathrm{AD}$ versus $\mathrm{VaD}$, $P=0.0060$. Vertical bars represent mean $\pm \mathrm{SE}$

DLB, $P=0.78$ for $\mathrm{AD}$, and $P=0.39$ for $\mathrm{VaD}$ ) or in all the groups combined $(P=0.75)$.

Myelin loss in the frontal lobes in dementing disorders as assessed by dMBP labelling

In the frontal $\mathrm{WM}$ of $\mathrm{VaD}$ cases, oligodendrocytic cell bodies of irregular size and myelin sheaths along the axon were densely stained for dMBP (Fig. 4a). dMBP-positive myelin sheaths were less frequently observed in $\mathrm{AD}$ and DLB compared to $\mathrm{VaD}$ but were still more frequent than in controls (Fig. 4a). Quantitative analysis showed that \%dMBP was significantly greater in $\mathrm{VaD}$ than in the other three groups (Fig. 4b). There was a significant inverse 

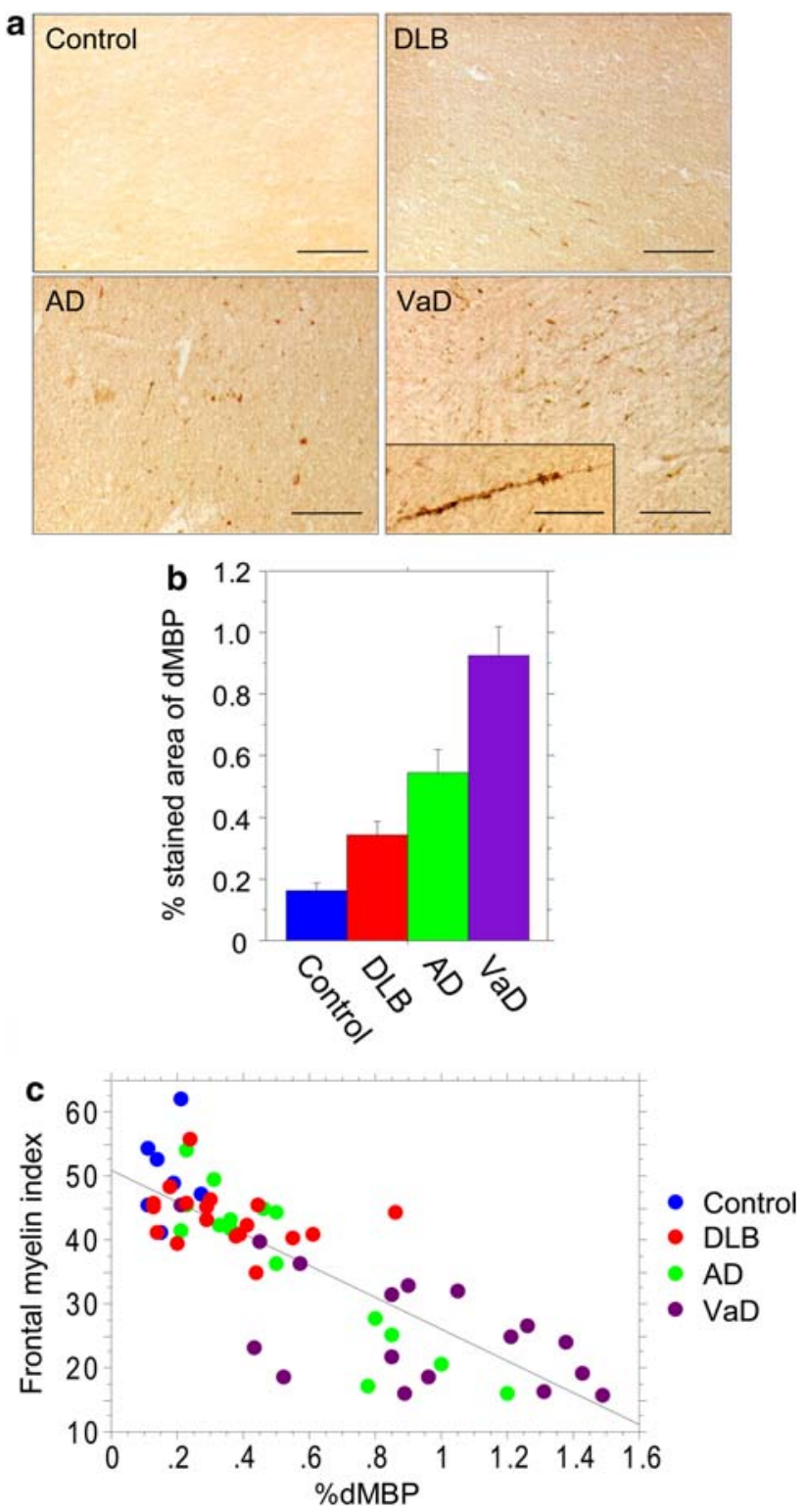

Fig. 4 Myelin damage in the frontal lobes in dementing disorders as assessed by novel dMBP labelling. a Representative images of dMBP staining in cases of control (89 years), AD (87 years), DLB (77 years), and $\mathrm{VaD}$ (78 years) (scale bars $100 \mu \mathrm{m}$ ). Inset in $\mathrm{VaD}$ shows degenerative dMBP-positive myelin sheaths along the axon (scale bar $50 \mu \mathrm{m})$. b \%dMBP in the four groups. Vertical bars represent mean $\pm \mathrm{SE}$. Differences were significant between any two groups: controls versus DLB, $P=0.0160$; controls versus $\mathrm{AD}$, $P=0.0029$; controls versus $\mathrm{VaD}, P<0.0001$; DLB versus $\mathrm{AD}$, $P=0.0331 ;$ DLB versus $\mathrm{VaD}, P<0.0001 ; \mathrm{AD}$ versus $\mathrm{VaD}$, $P=0.0044$. c An inverse correlation between the \%dMBP and the myelin index in the 59 cases combined $(r=-0.823, P<0.0001)$

correlation between the \% $\mathrm{dMBP}$ and the myelin index in all the 59 cases combined $(r=-0.823, P<0.0001)$ (Fig. 4c). Consistent with this, the \%dMBP positively correlated with the \%area for the first quartile of myelin density $(r=0.823$, $P<0.0001)$ and negatively correlated with that for the second quartile $(r=-0.777, P<0.0001)$.
Oligodendrocyte status with GSTpi immunohistochemistry

The \%dMBP was significantly but inversely correlated with the size of oligodendrocytes in $\mathrm{VaD}$ cases $(r=-0.70$, $P=0.0017 ; \quad r=-0.48, \quad P=0.023 ; \quad r=-0.72, \quad P=$ $0.0008 ; r=-0.76, P<0.0001 ; r=-0.79, P<0.0001)$ (Fig. 5a). This robust inverse correlation was also shown when all the five cases were combined $(r=-0.628$, $P<0.0001)$. In the areas for the first quartile where dMBP staining was extensive, a number of small, irregularly shaped oligodendrocytes were labelled for dMBP as well as GSTpi, indicating that damaged oligodendrocytes were shrunken (Fig. 5c). In two VaD cases, the \%dMBP significantly correlated with the density of oligodendrocytes (Fig. 5b, solid lines; $r=-0.64, P=0.0010 ; r=-0.54$, $P=0.025)$, while in other three $\mathrm{VaD}$ cases it did not reach statistical significance (Fig. 5b, dotted lines). In the infarcted area with macrophage infiltration, the GSTpipositive cells mostly disappeared, possibly reflecting loss of oligodendrocytes after severe ischaemia (Fig. 5c, rightmost panels).

By contrast, the \%dMBP did not correlate with size of oligodendrocytes in the four $\mathrm{AD}$ cases (Fig. 6a, dotted lines), but even positively correlated in one $\mathrm{AD}$ case ( $r=0.56, P=0.018$ ) (Fig. 6a, solid line). However, the $\%$ dMBP correlated with the density of oligodendrocytes in all five AD cases $(r=0.84, P<0.0001 ; r=0.68$, $P=0.004 ; r=0.82, P<0.0001 ; r=0.85, P<0.0001$; $r=0.87, P<0.0001$ ) (Fig. 6b), which was opposite to the trend in $\mathrm{VaD}$ cases. Oligodendrocytes were apparently not shrunken but the density of GSTpi-positive oligodendrocytes was increased (Fig. 6c). When all the five AD cases were combined, a significant correlation was shown between \%dMBP and the density of oligodendrocytes $(r=0.652, P<0.0001)$. A two-tier regression analysis showed that the absolute value of the $\mathrm{T}$ ratio (regression coefficient; SE) was highest for the 'disease' variable (disease code; $0=\mathrm{AD}$ and $1=\mathrm{VaD}$ ). This analysis showed the disease effects (VaD or $\mathrm{AD}$ ) mainly determine the relationship between the \%dMBP and the size or density of oligodendrocytes rather than age or gender across the cases. In terms of the 'size' of oligodendrocytes as an outcome variable, the deviance was the lowest (151.29) when only 'disease' was introduced to explanatory variables (coefficient, -2.45 ; SE, $0.40 ; P<0.001$ ), while in terms of the 'density' of oligodendrocytes, the deviance was the lowest (160.96) when both 'disease' (-708.0; $241.8 ; 0.023)$ and 'patient age' $(21.3 ; 8.95 ; 0.244)$ were introduced to them. No other parameter estimates reached statistical significance.

In the DLB cases, the results were variable: in one case, the size of oligodendrocytes was inversely correlated with 
Fig. 5 Correlation analysis between \%dMBP and density/ size of oligodendrocytes in the frontal lobe of $\mathrm{VaD}$ cases. a The $\%$ dMBP was inversely correlated with the size of oligodendrocytes (OLGs) with statistical significance (blue points, $r=-0.70$; red points, $r=-0.48 ;$ green points, $r=$ -0.72 ; light-blue points, $r=$ -0.76 ; brown points, $r=-0.79)$. Each point represents a ROI $(n=20-22)$ within WM per Fig. 3a. b The $\%$ dMBP inversely correlated with the density of oligodendrocytes in two $\mathrm{VaD}$ cases with statistical significance (red points, $r=-$ 0.64 ; brown points, $r=-0.54$ ) but not in the others. c Images for dMBP and GSTpi in two adjacent sections in a 78-yearold patient with $\mathrm{VaD}$, showing that increased myelin damage (from left to right panels) is accompanied by shrunken oligodendrocytes. The infarcted areas with dMBP-positive macrophages (arrows) as shown in the rightmost panels were excluded from the dMBP analysis. Magnified images of GSTpi-positive oligodendrocytes are shown in insets (scale bars $50 \mu \mathrm{m}$ )
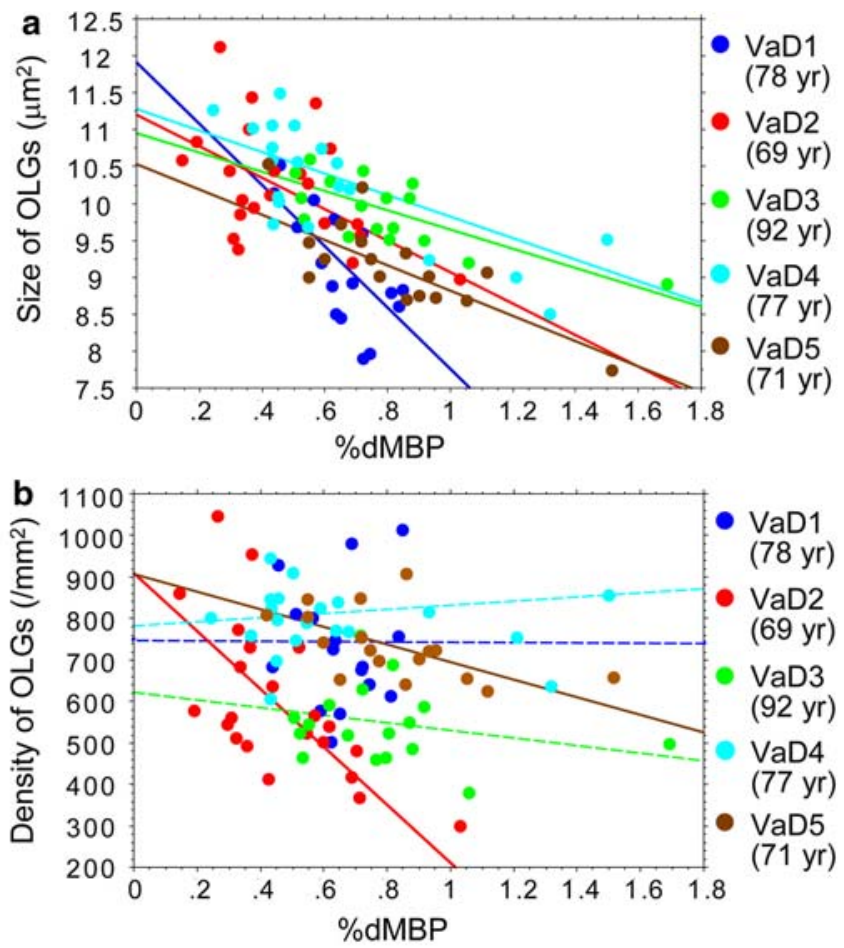

C

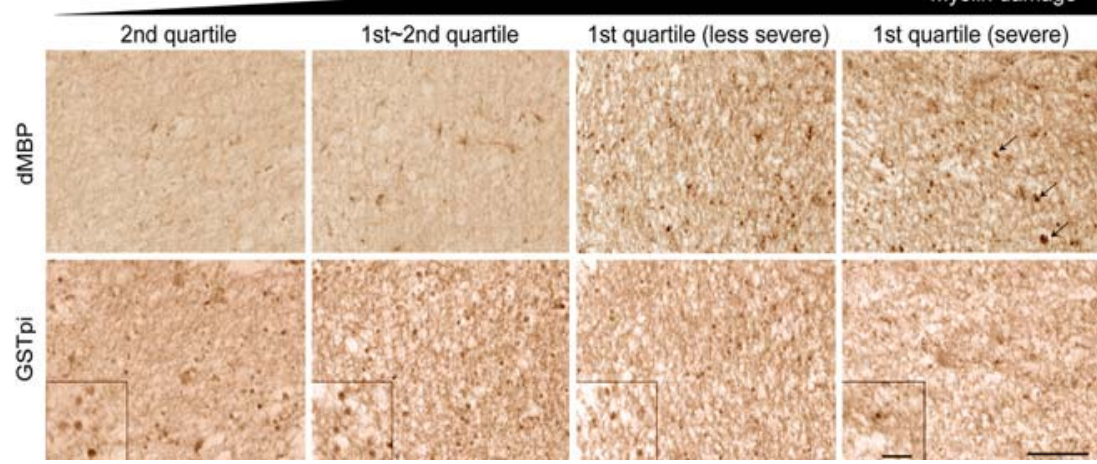

the \% dMBP as was the case with $\mathrm{VaD}$ but this was not so in the other two cases. The density of oligodendrocytes was also not correlated with \%dMBP (not shown).

\section{Discussion}

We utilized computer-assisted analysis to quantify myelin loss in brain tissues labelled with LFB and also developed a method to visualize damaged WM using the commercially available dMBP antibody [34, 47]. Myelin loss in the frontal lobes, as assessed by LFB and verified with $\mathrm{H} \& \mathrm{E}$ staining, was most severe in $\mathrm{VaD}$, less so in $\mathrm{AD}$ and $\mathrm{DLB}$, but still greater than in elderly controls. We also showed that frontal but not temporal WM changes appear as the pathological correlates of non-advanced dementia [7, 41, 48]. At more advanced stages of dementia, the temporal lobe WM exhibits more changes in all dementia types, particularly in AD, where axonal degeneration with secondary demyelination is likely more prominent [14]. Previous reports suggest that both the sclerotic changes in the medullary arteries and the WM changes in subcortical $\mathrm{VaD}$ are most prominent in the frontal lobe whereas least prominent in the temporal lobe [2, 19]. Thus, the frontal lobe may be the most susceptible to a hemodynamic derangement [22], leading to the severe WM changes, while the temporal lobe is less susceptible, resulting in smaller differences in temporal WM changes between $\mathrm{VaD}$ and the other groups. This is consistent with the previous imaging and pathological studies suggesting that frontal WM is preferentially vulnerable compared to the temporal WM during ageing and vascular disease [2, 41, 48].

We confirmed that dMBP could be used as an informative marker of myelin degeneration in cerebrovascular and neurodegenerative disorders [34, 47] and in rat models of chronic cerebral hypoperfusion [49]. Furthermore, 
Fig. 6 Correlation analysis between \%dMBP and density/ size of oligodendrocytes in the frontal lobe of $\mathrm{AD}$ cases. a The $\%$ dMBP did not significantly correlate with the size of oligodendrocytes (OLGs) in two $\mathrm{AD}$ cases but it did in one $\mathrm{AD}$ case $(r=0.56)$. Each point represents a ROI $(n=20-22)$ within WM per Fig. 3a. b The \%dMBP significantly correlated with the density of oligodendrocytes (blue points, $r=0.84$; red points, $r=0.68$; green points, $r=0.82$; lightblue points, $r=0.85$; brown points, $r=0.87)$. $\mathbf{c}$ Images for dMBP and GSTpi in two adjacent sections in an 87-yearold patient with $\mathrm{AD}$, showing that increased myelin damage (from left to right panels) is accompanied by increased density of oligodendrocytes (scale bars $50 \mu \mathrm{m}$ )
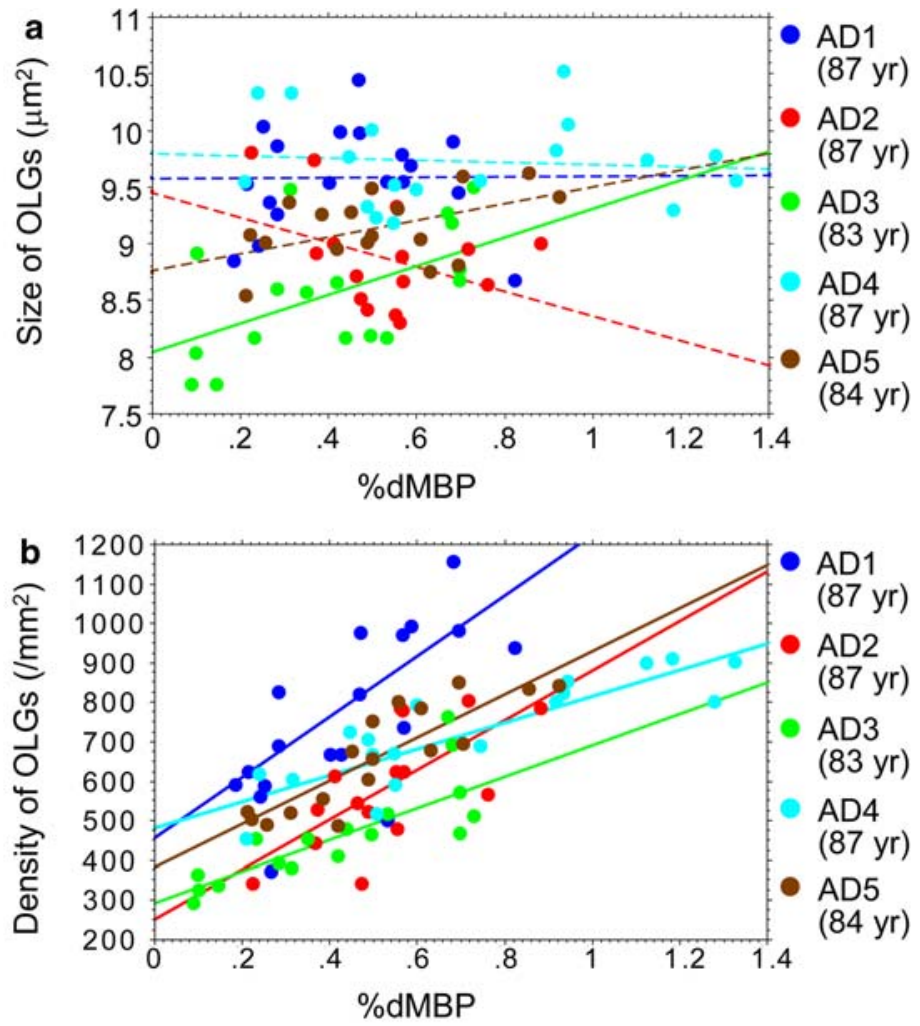

C Myelin damage

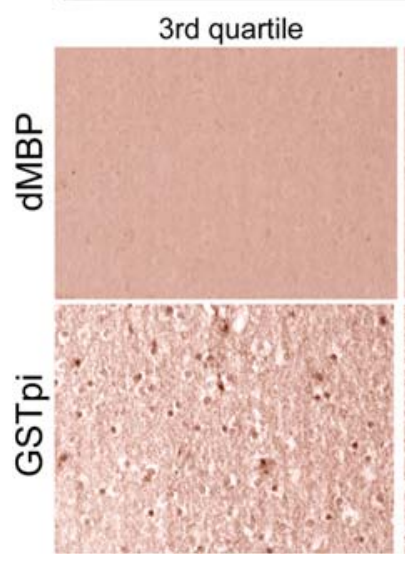
1st quartile

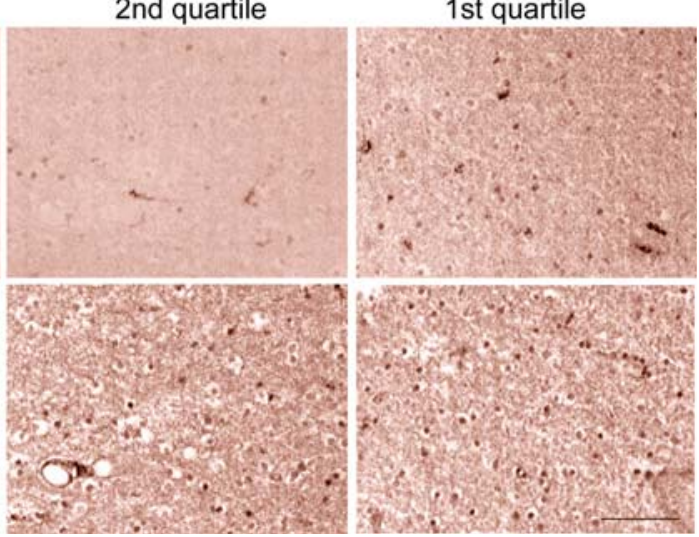

correlation analyses using adjacent sections labelled for dMBP or GSTpi suggest that differential mechanisms underlie $\mathrm{WM}$ damage between $\mathrm{VaD}$ and $\mathrm{AD}$. The WM damage may originate from hypoxic-ischaemic damage to oligodendrocytes in $\mathrm{VaD}$ whereas secondary to axonal degeneration in $\mathrm{AD}$, although the two pathologies at least partially overlap. Our observations on differential myelin loss between the dementias are also consistent with in vivo MR studies of WM lesions [5, 11].

We noted areas of severe $\mathrm{WM}$ changes even in $\mathrm{AD}$, i.e. the WM area that falls into the first quartile and the focal area with high \%dMBP, which suggests that severe hypoperfusive injury or oligaemia [17] could have occurred in these cases. However, severe ischaemia should lead to infarcts and decreased numbers of oligodendrocytes, which were not evident in the AD cases, partially because cases with co-existing $\mathrm{VaD}$ and $\mathrm{AD}$ were excluded from this study. A more plausible explanation is that the axons of the neocortical degenerating neurons also degenerate severely enough to provoke severe WM changes in AD [36]. These WM changes may be followed by recruitment of oligodendrocyte progenitor cells (OPCs) and their differentiation to mature oligodendrocytes $[45,50]$. Such a restorative mechanism may represent attempted remyelination of the WM, which could partially explain the differential degrees of $\mathrm{WM}$ damage between $\mathrm{AD}$ and $\mathrm{VaD}$. 
In contrast to the findings here, previous observations suggested that $\mathrm{WM}$ disease in $\mathrm{AD}$ entails reduction in number of oligodendrocytes [42, 43]. The conflicting results could be accounted for by the differences in patient selection or duration of disease severity, and the methodology used including different approaches to the computerassisted analyses [42, 43].

Our findings suggest that oligodendrocytes shrink because of ischaemia or oligaemia in the WM as even known from older morphological descriptions [39]. The shrinkage of oligodendrocytes may be a preliminary step towards cell death in $\mathrm{VaD}$. It is plausible that ischaemic injury in the WM involves a chronic hypoxic state [17] in $\mathrm{VaD}$ and that OPCs are damaged and do not retain the ability to compensate for the myelin loss. Consistent with this notion, in an unselected cohort of the elderly, remyelinating oligodendrocytes were reported to be lost in the centre of severely demyelinated WM but proliferated in the adjacent WM [41]. Similarly, in a rat experimental stroke model, the number of OPCs and mature remyelinating oligodendrocytes reduced in the ischaemic core but were increased in the periinfarct area [44]. These results suggest that severe ischaemia leads to unsuccessful remyelination as a result of impaired recruitment of OPCs, whereas mild to moderate ischaemia activates the myelin repair system. This may be comparable to multiple sclerosis, where oligodendrocytes become small and non-functional following long-lasting inflammatory insults with subsequent loss of myelin formation [11, 48].

Intriguingly, in one DLB case, an inverse correlation between \% dMBP and size of oligodendrocytes was evident in the frontal WM, suggesting that some of the cases bear underlying WM pathology of vascular origin, although a caution should be made on interpretation of data on the basis of a single DLB case. Aged brains, particularly the oldest ones have pathologies of vascular origin to a great extent [23]. Consistent with this, some of the non-demented elderly controls had focal areas of positive dMBP in the frontal WM. A retrospective study [32] had reported that frontal lobe WM pathology was more often evident in the clinically diagnosed DLB [31] than the AD patients but $80 \%$ of the DLB cases also exhibited mild to severe AD pathology [32]. In another study of 96 DLB cases, 38\% who had moderate to severe AD pathology also showed mild $(25 \%)$ to severe $(7 \%)$ cerebrovascular changes involving the WM [24]. These data collectively suggest that the neurodegenerative process in DLB is compounded by $\mathrm{AD}$ or vascular pathology.

A possible limitation of this study is that GSTpi is not the perfect marker of mature oligodendrocytes although we selected GSTpi over other currently used markers including 2',3'-cyclic nucleotide $3^{\prime}$-phosphodiesterase, MBP, myelin oligodendrocyte glycoprotein, Nogo-A [20] and
SRY-box containing gene 10 [4] because antibodies to GSTpi best labelled mature oligodendrocytes in a variety of human brain specimens using different antigen retrieval protocols and buffers. We believe biological variability from case to case may explain this since the marker perfectly labelled oligodendrocytes in non-human primate brain tissue (MI and RNK, unpublished observations) which was obtained under controlled perfusion and fixation conditions. Despite this, the evaluation of oligodendrocytes was validated by dMBP labelling since the antibody used was unique in detecting 'unhealthy' and damaged oligodendrocytes. We suggest that a combination of LFB staining with immunostaining for dMBP and GSTpi is a robust approach for analysing WM myelin loss and the pathogenesis of WM disease, which may also entail inflammatory/immune mechanisms [13].

In conclusion, our study emphasizes high frequency of frontal WM myelin loss in common dementing disorders, including $\mathrm{VaD}, \mathrm{AD}$, and $\mathrm{DLB}$, and enlightens the field with further clarification on myelin degradation $[12,16]$ and the differential status of oligodendrocytes. We suggest that myelin loss may evolve in parallel with oligodendrocytic activation in severely affected regions in AD but with shrunken oligodendrocytes consistent with a chronic hypoxic state [17] in VaD. Frontal WM changes have implications for attentional and executive impairments [33, $48]$ associated with fronto-subcortical circuits in dementing illness [10]. Our observations indicate that myelin loss in part occurs due to axonal degeneration [36], which needs to be similarly revisited across common dementing disorders.

Acknowledgments We thank Dr. A. Khundakar for critical reading of our manuscript and Ms. Shagufta Dalal for analysis of the myelin staining intensity. We are very grateful to the patients and families for their co-operation in the investigation of this study. M.I. was supported by overseas fellowships from the Kanae Foundation for the Promotion of Medical Science, the Mochida Memorial Foundation for Medical and Pharmaceutical Research, the Sumitomo Life Social Welfare Services Foundation, and the Daiwa Anglo-Japanese Foundation. Our work is supported by grants from the Medical Research Council (UK), the Alzheimer's Research Trust (UK) and the National Institutes of Health (NINDS).

Conflicts on Interest statement The authors do not have conflicts of interest related to this article.

Open Access This article is distributed under the terms of the Creative Commons Attribution Noncommercial License which permits any noncommercial use, distribution, and reproduction in any medium, provided the original author(s) and source are credited.

\section{References}

1. Akiguchi I, Tomimoto H, Suenaga T, Wakita H, Budka H (1997) Alterations in glia and axons in the brains of Binswanger's disease patients. Stroke 28:1423-1429 
2. Akiguchi I, Tomimoto H, Wakita $\mathrm{H}$ et al (2004) Topographical and cytopathological lesion analysis of the white matter in Binswanger's disease brains. Acta Neuropathol 107:563-570

3. Alafuzoff I, Thal DR, Arzberger T et al (2009) Assessment of beta-amyloid deposits in human brain: a study of the BrainNet Europe Consortium. Acta Neuropathol 117:309-320

4. Bannykh SI, Stolt CC, Kim J, Perry A, Wegner M (2006) Oligodendroglial-specific transcriptional factor SOX10 is ubiquitously expressed in human gliomas. J Neurooncol 76:115-127

5. Barber R, Scheltens P, Gholkar A et al (1999) White matter lesions on magnetic resonance imaging in dementia with Lewy bodies, Alzheimer's disease, vascular dementia, and normal aging. J Neurol Neurosurg Psychiatry 67:66-72

6. Bartzokis G, Sultzer D, Lu PH, Nuechterlein KH, Mintz J, Cummings JL (2004) Heterogeneous age-related breakdown of white matter structural integrity: implications for cortical "disconnection" in aging and Alzheimer's disease. Neurobiol Aging 25:843-851

7. Bozzali M, Falini A, Cercignani M et al (2005) Brain tissue damage in dementia with Lewy bodies: an in vivo diffusion tensor MRI study. Brain 128:1595-1604

8. Braak H, Alafuzoff I, Arzberger T, Kretzschmar H, Del Tredici K (2006) Staging of Alzheimer disease-associated neurofibrillary pathology using paraffin sections and immunocytochemistry. Acta Neuropathol 112:389-404

9. Braak H, Braak E (1991) Neuropathological stageing of Alzheimer-related changes. Acta Neuropathol 82:239-259

10. Burton EJ, Kenny RA, O'Brien J et al (2004) White matter hyperintensities are associated with impairment of memory, attention, and global cognitive performance in older stroke patients. Stroke 35:1270-1275

11. Burton EJ, McKeith IG, Burn DJ, Firbank MJ, O’Brien JT (2006) Progression of white matter hyperintensities in Alzheimer disease, dementia with Lewy bodies, and Parkinson disease dementia: a comparison with normal aging. Am J Geriatr Psychiatry $14: 842-849$

12. Connor JR (2004) Myelin breakdown in Alzheimer's disease: a commentary. Neurobiol Aging 25:45-47

13. Duce JA, Hollander W, Jaffe R, Abraham CR (2006) Activation of early components of complement targets myelin and oligodendrocytes in the aged rhesus monkey brain. Neurobiol Aging 27:633-644

14. Englund E (1998) Neuropathology of white matter changes in Alzheimer's disease and vascular dementia. Dement Geriatr Cogn Disord 9(Suppl 1):6-12

15. Englund E (2000) Neuropathology of white matter disease: parenchymal changes. In: ID Pantoni L, Wallin A (eds) The Matter of white matter. Clinical and pathophysiological aspects of white matter disease related to cognitive decline and vascular dementia, vol 10. Academic Pharmaceutical Productions, Utrecht, pp 223-246

16. Erkinjuntti T, Benavente O, Eliasziw M et al (1996) Diffuse vacuolization (spongiosis) and arteriolosclerosis in the frontal white matter occurs in vascular dementia. Arch Neurol 53:325-332

17. Fernando MS, Simpson JE, Matthews F et al (2006) White matter lesions in an unselected cohort of the elderly: molecular pathology suggests origin from chronic hypoperfusion injury. Stroke 37:1391-1398

18. Firbank MJ, Minett T, O'Brien JT (2003) Changes in DWI and MRS associated with white matter hyperintensities in elderly subjects. Neurology 61:950-954

19. Furuta A, Ishii N, Nishihara Y, Horie A (1991) Medullary arteries in aging and dementia. Stroke 22:442-446

20. Gil V, Nicolas O, Mingorance A et al (2006) Nogo-A expression in the human hippocampus in normal aging and in Alzheimer disease. J Neuropathol Exp Neurol 65:433-444
21. Gouw AA, Seewann A, Vrenken H et al (2008) Heterogeneity of white matter hyperintensities in Alzheimer's disease: post-mortem quantitative MRI and neuropathology. Brain 131:3286-3298

22. Haglund M, Kalaria R, Slade JY, Englund E (2006) Differential deposition of amyloid beta peptides in cerebral amyloid angiopathy associated with Alzheimer's disease and vascular dementia. Acta Neuropathol 111:430-435

23. Jellinger KA (2007) The enigma of vascular cognitive disorder and vascular dementia. Acta Neuropathol 113:349-388

24. Jellinger KA (2003) Prevalence of vascular lesions in dementia with Lewy bodies. A postmortem study. J Neural Transm 110:771-778

25. Kalaria R (2002) Similarities between Alzheimer's disease and vascular dementia. J Neurol Sci 203-204:29-34

26. Kalaria RN (1996) Cerebral vessels in ageing and Alzheimer's disease. Pharmacol Ther 72:193-214

27. Kalaria RN, Hedera P (1995) Differential degeneration of the cerebral microvasculature in Alzheimer's disease. Neuroreport $6: 477-480$

28. Kalaria RN, Kenny RA, Ballard CG, Perry R, Ince P, Polvikoski $\mathrm{T}$ (2004) Towards defining the neuropathological substrates of vascular dementia. J Neurol Sci 226:75-80

29. Koga H, Yuzuriha T, Yao H et al (2002) Quantitative MRI findings and cognitive impairment among community dwelling elderly subjects. J Neurol Neurosurg Psychiatry 72:737-741

30. Lewis H, Beher D, Cookson N et al (2006) Quantification of Alzheimer pathology in ageing and dementia: age-related accumulation of amyloid-beta(42) peptide in vascular dementia. Neuropathol Appl Neurobiol 32:103-118

31. Londos E, Passant U, Brun A, Gustafson L (2000) Clinical Lewy body dementia and the impact of vascular components. Int J Geriatr Psychiatry 15:40-49

32. Londos E, Passant U, Gustafson L, Brun A (2001) Neuropathological correlates to clinically defined dementia with Lewy bodies. Int J Geriatr Psychiatry 16:667-679

33. Makris N, Papadimitriou GM, van der Kouwe A et al (2007) Frontal connections and cognitive changes in normal aging rhesus monkeys: a DTI study. Neurobiol Aging 28:1556-1567

34. Matsuo A, Lee GC, Terai K et al (1997) Unmasking of an unusual myelin basic protein epitope during the process of myelin degeneration in humans: a potential mechanism for the generation of autoantigens. Am J Pathol 150:1253-1266

35. McKeith IG, Dickson DW, Lowe J et al (2005) Diagnosis and management of dementia with Lewy bodies: third report of the DLB Consortium. Neurology 65:1863-1872

36. Medana IM, Esiri MM (2003) Axonal damage: a key predictor of outcome in human CNS diseases. Brain 126:515-530

37. Mirra SS (1997) The CERAD neuropathology protocol and consensus recommendations for the postmortem diagnosis of Alzheimer's disease: a commentary. Neurobiol Aging 18:S91S94

38. Pantoni L, Garcia JH (1997) Pathogenesis of leukoaraiosis: a review. Stroke 28:652-659

39. Pantoni L, Garcia JH (1995) The significance of cerebral white matter abnormalities 100 years after Binswanger's report. A review. Stroke 26:1293-1301

40. Premkumar DR, Cohen DL, Hedera P, Friedland RP, Kalaria RN (1996) Apolipoprotein E-epsilon4 alleles in cerebral amyloid angiopathy and cerebrovascular pathology associated with Alzheimer's disease. Am J Pathol 148:2083-2095

41. Salat DH, Tuch DS, Greve DN et al (2005) Age-related alterations in white matter microstructure measured by diffusion tensor imaging. Neurobiol Aging 26:1215-1227

42. Sjobeck M, Englund E (2003) Glial levels determine severity of white matter disease in Alzheimer's disease: a neuropathological study of glial changes. Neuropathol Appl Neurobiol 29:159-169 
43. Sjobeck M, Haglund M, Englund E (2005) Decreasing myelin density reflected increasing white matter pathology in Alzheimer's disease-a neuropathological study. Int $\mathrm{J}$ Geriatr Psychiatry 20:919-926

44. Tanaka K, Nogawa S, Suzuki S, Dembo T, Kosakai A (2003) Upregulation of oligodendrocyte progenitor cells associated with restoration of mature oligodendrocytes and myelination in periinfarct area in the rat brain. Brain Res 989:172-179

45. Tansey FA, Cammer W (1991) A pi form of glutathione- $S$ transferase is a myelin- and oligodendrocyte-associated enzyme in mouse brain. J Neurochem 57:95-102

46. Thal DR, Rub U, Orantes M, Braak H (2002) Phases of A betadeposition in the human brain and its relevance for the development of AD. Neurology 58:1791-1800
47. Tomimoto H, Akiguchi I, Matsuo A et al (1997) Encephalitogenic peptide (EP) in human cerebrovascular white matter lesions. Neuroreport 8:3727-3730

48. Tullberg M, Fletcher E, DeCarli C et al (2004) White matter lesions impair frontal lobe function regardless of their location. Neurology 63:246-253

49. Wakita H, Tomimoto H, Akiguchi I et al (2002) Axonal damage and demyelination in the white matter after chronic cerebral hypoperfusion in the rat. Brain Res 924:63-70

50. Wolswijk G (2000) Oligodendrocyte survival, loss and birth in lesions of chronic-stage multiple sclerosis. Brain 123(Pt 1):105-115

51. Yamamoto Y, Ihara M, Tham C et al (2009) Neuropathological correlates of temporal pole white matter hyperintensities in CADASIL. Stroke 40:2004-2011 